\title{
AEROMAGNETIC MAP OF NORTHWESTERN ONTONAGON COUNTY, MICHIGAN
}

GEOPHYSICAL INVESTIGATIONS

MAP GP- 834 